AGH DRILLING, OIL, GAS • Vol. $31 \cdot$ No. $2 \cdot 2014$

http://dx.doi.org/10.7494/drill.2014.31.2.325

\author{
Jacek Blicharski*, Czeslaw Rybicki*, Grzegorz Stec*
}

\title{
ANALYTICAL PREDICTION MODEL OF UGS PERFORMANCE
}

\section{INTRODUCTION}

Underground gas storages (UGS) play more and more prominent role in the energy security programs of numerous European countries, Poland including. The construction of high capacity underground gas storages, providing gas for the entire system, and local ones, became a very important element of energetic independence. Underground gas storages are one of the basic components of correctly operating gas systems providing fluent and flawless gas deliveries to the customers. The major objective of underground gas storages is compensating for seasonal oscillations in natural gas consumption.

Apart from compensating for seasonal oscillations in natural gas consumption, UGS also play the following functions $[9,10]$ :

- exploitation of a gaseous system with steady gas volumes, achieved thanks to the high coefficient of system utilization, and the resulting lower cost of gas transport,

- guaranteed full and uninterrupted gas deliveries to customers,

- continuous production from natural gas fields,

- plausible withdrawal of constant quantities of import gas,

- creation of economic and strategic gas reserves in the case of interrupted deliveries of import gas.

The present storage capacities in Poland do not fully suffice to regulate gas demand. Neither they can be treated as a reserve in the case of a long-term suspended gas import in winter. Therefore, the existing storages should be developed or new ones construed.

The depleted gas reservoirs are geologic objects which are most frequently used as underground gas storages. Among the main advantages of this type of structure are its proved

* AGH University of Science and Technology, Faculty of Drilling, Oil and Gas, Krakow, Poland 
tightness, appropriate pore volume, well recognized reservoir and thermodynamic characteristic and parameters of fluids. Moreover, these objects have a surface infrastructure which at the stage of performing the storage can be used for gas storing purposes.

The basic parameters of storage operation are active capacity, range of working pressure in the storage, daily gas withdrawal capacity and the related number of wells $[6,8]$.

An analytical mathematical model based on equations for well inflow, flow capacity and mass balance allowing for the analysis of UGS was worked out in the paper. The presented model was used in the aspect of maximized active capacity and efficiency of natural gas withdrawal from UGS for a given field/well configuration. Calculation variants were presented on the basis of calculated gas storage operation through vertical or horizontal wells. The calculations were performed for one of the gas fields in the south-eastern Poland.

\section{MATHEMATICAL MODEL OF UGS OPERATION}

The operation of an underground gas storage localized in a depleted reservoir lies in a cyclic injection and withdrawal of gas. The injection and withdrawal efficiency is a function of total drop of pressure difference between formation pressure and wellhead pressure and depends on the properties of the porous medium in which the flow takes place, extent to which the near-well zone has been damaged, properties of the extracted fluid, well design and character of flow in the interaction zone of the well and in the production pipes.

The production rate of a well is determined for the actual reservoir pressure and given wellhead pressure values by solving a system of equations for gas inflow and equation for flow capacity of well.

Gas inflow to the well is most frequently described by the so-called bi-term formula $[1,5]$ :

$$
p_{r}{ }^{2}-p_{w}{ }^{2}=a \cdot q+b \cdot q^{2}
$$

where $\mathrm{a}$ and $\mathrm{b}$ are defined by the following dependences

$$
a=\frac{\mu \cdot p_{n} \cdot z \cdot T}{\pi \cdot k \cdot h \cdot T_{n}} \cdot\left(\ln \frac{r_{e}}{r_{w}}-\frac{3}{4}+S_{m}+D_{t} \cdot q\right)
$$

and

$$
b=\frac{\mu \cdot p_{n} \cdot z \cdot T}{\pi \cdot k \cdot h \cdot T_{n}} \cdot D_{t}
$$

The turbulent flow coefficient $\mathrm{D}_{\mathrm{t}}$ can be calculated from the empirical dependence $[3,5]$ :

$$
D_{t}=\frac{6 \cdot 10^{-5} \cdot S_{g} \cdot k_{s}^{-0.1} \cdot h}{\mu \cdot r_{w} \cdot h_{p e r f}^{2}}
$$

The expression (4) is an empirical dependence where the permeability coefficient $\mathrm{f}$ is expressed in $\mathrm{mD}$, thickness $\mathrm{h}$ in $\mathrm{ft}$, well radius $r_{w}$ in $\mathrm{ft}$, thickness of perforation $\mathrm{h}_{\text {perf }}$ in $\mathrm{ft}$, whereas the coefficient of flow turbulence $\mathrm{D}_{\mathrm{t}}$ is in $[\mathrm{d} / \mathrm{Mscf}]$. 
The gas inflow to the well may be described with the Joshi equation [2]:

$$
q=\frac{\pi \cdot k_{H} \cdot h \cdot T_{n} \cdot\left(p_{r}{ }^{2}-p_{w}{ }^{2}\right)}{\mu \cdot p_{n} \cdot z \cdot T \cdot\left(\ln \left(\frac{a_{H}+\sqrt{a_{H}{ }^{2}-(L / 2)}}{L / 2}\right)+\frac{I_{a n i} \cdot h}{L} \cdot\left(\ln \frac{I_{a n i} \cdot h}{r_{w} \cdot\left(I_{a n i}+1\right)}-\frac{3}{4}+D_{t H} \cdot q\right)\right)}
$$

$I_{\text {ani }}$ is the coefficient of permeability anisotropy in the form:

$$
I_{a n i}=\sqrt{\frac{k_{H}}{k_{V}}}
$$

where: $\mathrm{k}_{\mathrm{H}}$ - coefficient of horizontal permeability $\mathrm{k}_{\mathrm{H}}$ is defined as geometrical average of directional permeabilities $k_{H}=\sqrt{k_{x} \cdot k_{y}}$, and vertical permeability as $k_{V}=k_{z}$, parameter , $\mathrm{a}_{\mathrm{H}}$ " in eq. (5) is half of the length of the longest axis of drainage ellipsoid of horizontal well. It is described with the following dependence:

$$
a_{H}=\frac{L}{2} \cdot\left(0.5+\left(0.25+\left(\frac{2 \cdot r_{e H}}{L}\right)^{4}\right)^{0.5}\right)^{0.5} \text { for } L / 2<0.9 \cdot r_{e H}
$$

The turbulent flow coefficient $\mathrm{D}_{\mathrm{tH}}$ in equation (5) can be described with the dependence

$$
D_{t H}=\frac{2.22 \cdot 10^{-15} \cdot S_{g} \cdot\left(k_{x} \cdot k_{y} \cdot k_{z}\right)^{1 / 3}}{\mu \cdot h \cdot r_{w H}} \cdot \beta_{H}
$$

Where: $\beta_{\mathrm{H}}$ - non-Darcy flow coefficient for a horizontal well can be determined from a correlation proposed by Tek

$$
\beta_{H}=\frac{5.5 \cdot 10^{9}}{\left(k_{x} \cdot k_{y} \cdot k_{z}\right)^{5 / 12} \cdot \phi^{3 / 4}}
$$

whereas the effective radius of a horizontal well $\mathrm{r}_{\mathrm{wH}}$ is defined by

$$
r_{w H}=\frac{r_{w} \cdot\left(1+I_{a n i}\right)}{2 \cdot I_{a n i}}
$$

Equations (8) and (9) are empirical dependences expressed in British units, i.e. gas viscosity in (cP), permeability in (mD), non-Darcy flow coefficient for horizontal well $\beta_{\mathrm{H}}$ in (1/ $\mathrm{ft}$ ), and turbulent flow coefficient $\mathrm{D}_{\mathrm{tH}}$ in $(\mathrm{d} / \mathrm{Mscf})$.

Gas flow in a well is described by well's flow capacity plot (curve VLP) defined on the basis of an equation for well's flow capacity, which for a vertical well with production pipes of constant cross-section, can be written as $[1,6]$ : 


$$
p_{w}{ }^{2}=p_{g}{ }^{2} \cdot e^{\frac{2 \cdot g \cdot H}{z \cdot R \cdot T}}+\frac{8 \cdot q^{2} \cdot p_{n}{ }^{2} \cdot z^{2} \cdot T^{2} \cdot \lambda}{\pi^{2} \cdot D^{5} \cdot T_{n}^{2} \cdot g} \cdot\left(e^{\frac{2 \cdot g \cdot H}{z \cdot R \cdot T}}-1\right)
$$

The mass balance equation described a change of pressure in a reservoir in a function of cumulative mass of extracted/injected gas can be described for a bulk gas reservoir by the following dependence

$$
\frac{p}{z}=\frac{P i}{z i} \cdot\left(1-\frac{G p-G z}{G}\right)
$$

where:

$$
\begin{aligned}
P_{i} & - \text { initial pressure, } \\
P & - \text { current pressure, } \\
G_{p} & - \text { amount of extracted gas, } \\
G & - \text { resources in a gas field. }
\end{aligned}
$$

\section{MODEL OF UNDERGROUND GAS STORAGE OPERATION}

The plausible active capacity and maximum gas withdrawal rate for an exemplary gas storage created in a depleted gas reservoir were assessed on the basis of the presented mathematical model. The analysis was made for a gas field in the Carpathian Forefield. This was a high-methane gas field. The gas composition was presented in table 1. Originally the gas resources equaled to $5.6 \mathrm{mld} \mathrm{Nm}^{3}$, and the initial pressure was $15.24 \mathrm{MPa}$. The analyzed field was a multihorizon, laminar reservoir of surface equal to $7.57 \mathrm{~km}^{2}$. The average effective thickness of the horizon was $75.5 \mathrm{~m}$. Lithologically, the reservoir consisted of sandstones of average porosity of $23 \%$ and permeability of $95 \mathrm{mD}$. The observation of the horizon revealed that formation fluids were underlying the horizon at nearly all its surface giving them a mass reservoir character. The analyses of production data revealed that the activity of these waters was small therefore gas expansion turned out to be the main source of reservoir energy. The reservoir was opened out with 6 vertical wells. The productivity rates of the wells were assessed on the basis of hydrodynamic tests. The initial potential productivity rates of the wells were about $4000 \mathrm{Nm}^{3} / \mathrm{min}$, and the admissible rates were established for $150-200 \mathrm{Nm}^{3} / \mathrm{min}$. The basic reservoir and well parameters are presented in table 2 .

Over $80 \%$ of initial resources have been extracted so far, therefore establishing an underground gas storage in that place would necessitate a buffer capacity.

Table 1

Composition of gas from the analyzed field

\begin{tabular}{|l|l|l|l|l|l|l|l|l|l|l|l|}
\hline Component & $\mathrm{C} 1$ & $\mathrm{C} 2$ & $\mathrm{C} 3$ & i-C4 & n-C4 & i-C5 & n-C5 & C6 & H2 & N2 & CO2 \\
\hline Participation [\%] & 98.8 & 0.31 & 0.13 & 0.1 & 0.04 & 0.06 & 0.01 & 0.03 & 0.01 & 0.50 & 0.01 \\
\hline
\end{tabular}


Table 2

Parameters of reservoir and wells

\begin{tabular}{|l|l|}
\hline Initial pressure & $15.24 \mathrm{MPa}$ \\
\hline Initial gas in place & $5.58 \mathrm{mld} \mathrm{Nm}^{3}$ \\
\hline Surface area of gas field & $7.57 \mathrm{~km}^{2}$ \\
\hline Average thickness of gas field & $75.5 \mathrm{~m}$ \\
\hline Average porosity & $23 \%$ \\
\hline Horizontal permeability & $95 \mathrm{mD}$ \\
\hline Reservoir temperature & $330 \mathrm{~K}$ \\
\hline Average depth of reservoirion & $1660 \mathrm{~m}$ \\
\hline Average diameter of production pipes (vertical well) & $3.5 \mathrm{in}$ \\
\hline Well radius & $0.15 \mathrm{~m}$ \\
\hline
\end{tabular}

\section{METHODICS}

The McVay and Spivey methodics [7] was adapted for determining the optimum active capacity of the planned storage. The constant gas injection and withdrawal rates from a storage of constant pore volume and predefined number of wells were assumed.

The first step was preparing the reservoir model with the predefined number of wells in a given configuration. Then the mass balance equation (12) was used and a gas in place vs. average reservoir pressure plot was worked out (fig. 1). Owing to the bulk character of the reservoir, the relation was linear. The maximum formation pressure should not exceed the initial pressure, which corresponds to the initial gas in place.

For determining the active and buffer capacity of a storage it was necessary of work out a well productivity plot for the injection and withdrawal periods. The well productivity plot was based on the nodal analysis working out the gas in place (IPR) plot for a given formation pressure and flow capacity of well (VLP) for the assumed wellhead pressure. The point of intersection defined the proper productivity rate of the well. By changing the reservoir pressure the point of intersection of IPR and VLP curves gave a new productivity rate of the well [1] (fig. 2). By interpolating the results on the plot in the well productivity rate vs. average reservoir pressure coordinates system we got the production rate of well for the injection and withdrawal periods (figs. 3).

The well production rate curve for the withdrawal period was worked out on the basis of the production rate in the course of gas withdrawal from UGS at a given minimum wellhad pressure. With the increase of the quantity of produced gas the reservoir pressure decreased, thus the production rate of the well deteriorated as well. Therefore each reservoir pressure had its maximum well production rate and gas in place. As visualized in figure 3 , the well productivity curve for the period of gas withdrawal was plotted for minimum wellhead pressure of 30 bar. The productivity curve of the same well for the injected gas was plotted for 
the maximum wellhead pressure value, i.e. 150 bar. During gas injection to UGS the reservoir pressure increased and the injection rate was observed to decrease. A reverse dependance was noted for gas withdrawal. With the increasing reservoir pressure values the withdrawal rate went up, too.

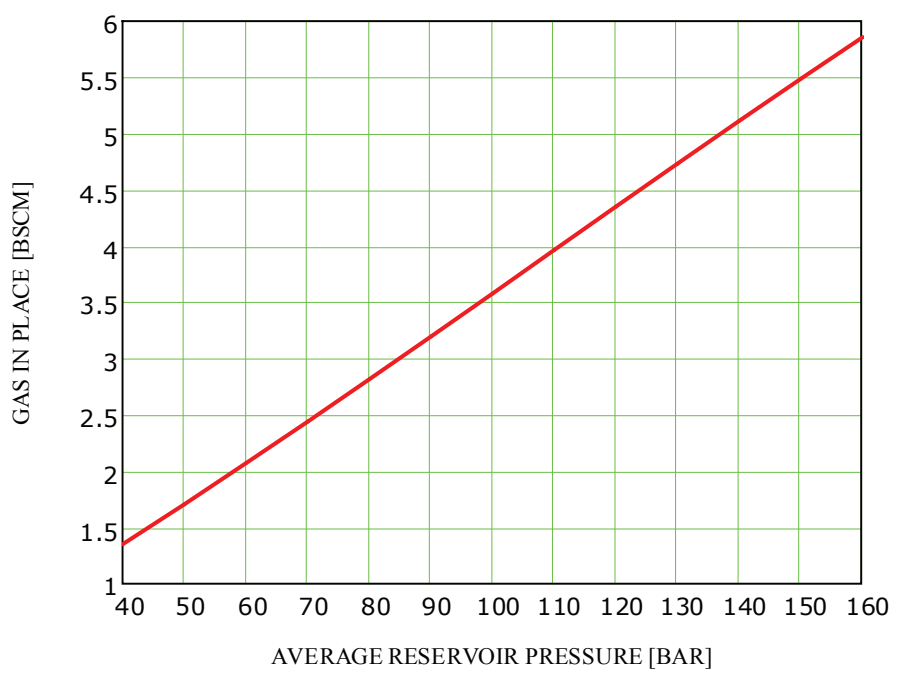

Fig. 1. Amount of gas in the storage in a function of average reservoir pressure [own adaptation]

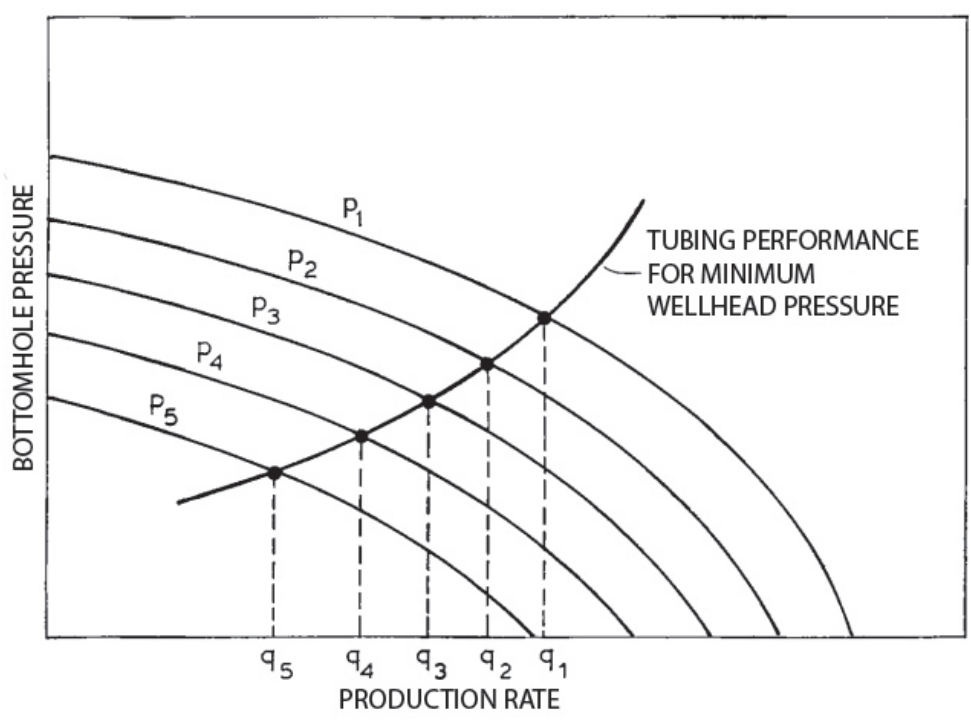

Fig. 2. Nodal analysis - determining productivity of a well [1] 


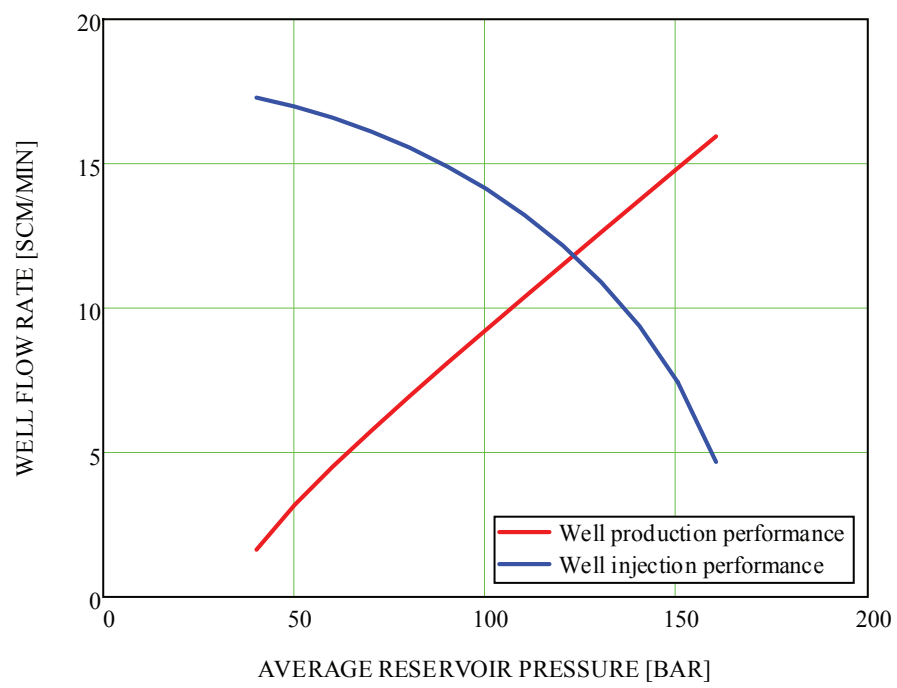

Fig. 3. Well production rate plots [own adaptation]

\section{DETERMINING THE UGS PRESSURES- ACTIVE AND BUFFER CAPACITIES}

Having assumed that a given reservoir pressure equals to the pressure observed at the end of the gas withdrawal phase, one may calculate the amount of produced gas and so determine gas in place and the corresponding reservoir pressure from the known production rate of a well for this pressure, number of wells and time of gas withdrawal from UGS. The determined pressure was the required reservoir pressure at the beginning of the withdrawal cycle, thanks to which the gas could be extracted at a constant rate (determined for a pressure at the end of the withdrawal stage) over the entire period of gas withdrawal. Performing similar calculations for the successive reservoir pressures we were given demanded pressure at the beginning of the withdrawal stage. On this basis the dependance of average reservoir pressure at the end and at the beginning of the gas withdrawal cycle was plotted (fig. 4).

A similar procedure was performed for gas injection cycle. Having assumed a certain value of maximum injection pressure on wellhead, the gas injection rate was determined for various reservoir pressure values on the basis of nodal analysis. The obtained plot was the well production rate plot (fig. 3).

Having assumed a certain value of UGS pressure at the end of the gas injection stage (e.g. initial reservoir pressure), the injection flow rate could be calculated for that pressure from the well production rate curve. Based on the known number of wells and time of injection, the total amount of injected gas was determined. After subtracting the quantity of injected gas in UGS from the gas in place at the end of the withdrawal stage 
we got the amount of gas in UGS and the corresponding pressure at the beginning of the injection stage. Pressure and gas in place values at the beginning of the injection stage were obtained after making recalculations for the successive pressures at the end of the injection stage. A plot representing dependances between average reservoir pressure at the beginning and at the end of the withdrawal stage was worked out on the basis of these calculations (fig. 4).

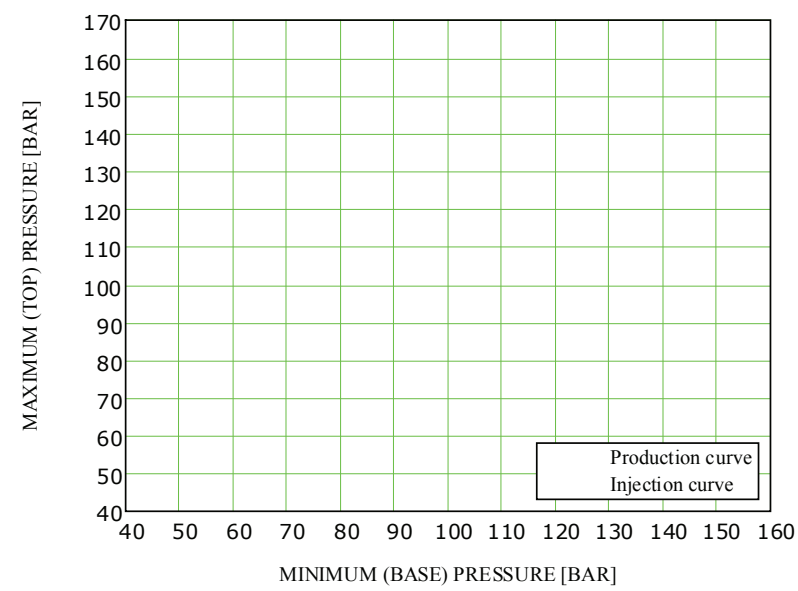

Fig. 4. Determining optimum range of pressures for UGS operation [own adaptation]

By superimposing the results of pressure calculations at the beginning and at the end stage of injection and withdrawal cycles onto one common plot a point of intersection was found out. This point defined the range of minimum and maximum storage pressure (fig. 4). On this basis the buffer and active capacities were established. The buffer capacity corresponded to the quantity of gas in the storage at minimum pressure, whereas active capacity was a difference of gas quantity at maximum and minimum pressures.

\section{RESULTS OF CALCULATIONS AND THEIR ANALYSIS}

The objective of this analysis was modeling of UGS operation in the aspect of maximized active capacity and withdrawal efficiency of UGS through a given number of vertical and horizontal wells.

To simplify the evaluation of the obtained results, the same time of injection and withdrawal was assumed (120 days). Two values of minimum wellhead pressure at the gas withdrawal stage were assumed in the calculations, i.e. 30 and 60 bar. The maximum wellhead pressure of gas injection was established for 150 bar. It was also assumed that the maximum UGS pressure could slightly exceed the initial reservoir pressure.

The production rate of vertical wells was defined by equation (3) on the basis of the results of hydrodynamic tests performed in one of the wells at the initial stage of gas production. 
The assumed values $\mathrm{a}$ and $\mathrm{b}$ in eq. (3) equaled to $\mathrm{a}=2.95 \mathrm{bar}^{2} \cdot \mathrm{min} / \mathrm{m}^{3}$ and $\mathrm{b}=0.00086$ $\mathrm{bar}^{2} \cdot \mathrm{min}^{2} / \mathrm{m}^{6}$.

The production rate of horizontal wells was determined with the use of equation (6). The anisotropy of permeability of reservoir was assumed, i.e. the permeability coefficient in vertical direction as $1 / 3$ of horizontal permeability. The horizontal section was assumed to be $500 \mathrm{~m}$ long and the production pipes 5 " in diameter.

\section{STORING WITH THE USE OF VERTICAL WELLS}

The results of calculations for the UGS with the use of vertical wells are visualized in table 3 and figs. 5 to 8 .

Table 3

Results of calculations for storages making use of vertical wells

\begin{tabular}{|c|c|c|c|c|c|c|}
\hline Number of wells & 10 & 20 & 30 & 40 & 50 & 60 \\
\hline \multicolumn{7}{|l|}{ Wellhead Pressure 30 bar } \\
\hline Base Gas Capacity, [Bscm] & 3.88 & 3.42 & 3.06 & 2.76 & 2.53 & 2.35 \\
\hline Working Gas Capacity, [Bscm] & 1.05 & 1.82 & 2.39 & 2.81 & 3.14 & 3.39 \\
\hline Well Withdrawal Rate, $[\mathrm{Scm} / \mathrm{sec}]$ & 10.13 & 8.77 & 7.68 & 6.77 & 6.04 & 5.44 \\
\hline UGS Withdrawal Rate, [Mln Scm/d] & 8.75 & 15.14 & 19.9 & 23.4 & 26.1 & 28.2 \\
\hline Top Reservoir Pressure, [bar] & 135.1 & 143.2 & 148.8 & 152.3 & 154.7 & 156.6 \\
\hline Base Reservoir Pressure, [bar] & 107.8 & 95.79 & 86.37 & 78.66 & 72.49 & 67.56 \\
\hline Depression $\left(\mathrm{P}_{\mathrm{r}}-\mathrm{P}_{\mathrm{w}}\right)$, [bar] & 9.87 & 9.45 & 9.06 & 8.68 & 8.32 & 7.98 \\
\hline \multicolumn{7}{|l|}{ Wellhead Pressure 60 bar } \\
\hline Base Gas Capacity, [Bscm] & 4.16 & 3.76 & 3.47 & 3.25 & 3.08 & 2.96 \\
\hline Working Gas Capacity, [Bscm] & 0.97 & 1.64 & 2.12 & 2.45 & 2.70 & 2.90 \\
\hline Well Withdrawal Rate, $[\mathrm{Scm} / \mathrm{sec}]$ & 9.31 & 7.91 & 6.81 & 5.92 & 5.22 & 4.67 \\
\hline UGS Withdrawal Rate, [Mln Scm/d] & 8.05 & 13.66 & 17.65 & 20.46 & 22.54 & 24.19 \\
\hline Top Reservoir Pressure, [bar] & 140.2 & 147.6 & 152.6 & 155.7 & 158.0 & 160.0 \\
\hline Base Reservoir Pressure, [bar] & 115.0 & 104.7 & 97.17 & 91.40 & 87.10 & 83.90 \\
\hline Depression $\left(\mathrm{P}_{\mathrm{r}}-\mathrm{P}_{\mathrm{w}}\right)$, [bar] & 8.32 & 7.59 & 6.92 & 6.30 & 5.75 & 5.29 \\
\hline
\end{tabular}

Figure 5 illustrates how active and buffer capacities change in a function of the vertical wells number at minimum wellhead pressure of 30 and 60 bar, respectively.

With the increasing number of wells the active capacity of UGS increases and the demanded buffer capacity decreases. Those results are strongly influenced by the minimum 
wellhead pressure values. At the lower injection wellhead pressures the active capacity increases and the buffer capacity decreases.

The magnitude of active and buffer capacities is directly connected with the range of UGS pressures, as in fig. 6. Depending on the number of wells and minimum wellhead pressure, the difference between minimum and maximum pressures in the UGS increases; at the same time the maximum UGS pressure increases for higher wellhead pressure values.

The highest active capacity of UGS at a level of $3.4 \mathrm{mld} \mathrm{Nm}^{3}$ was obtained for a wellhead pressure 30 bar with the use of 60 vertical wells. The storage production rate at the final stage of withdrawal was $24 \mathrm{mln} \mathrm{Nm}^{3} / \mathrm{d}$ in this variant.

The changes in the UGS gas withdrawal rate and withdrawal rate with one well in a function of the number of wells are visualized in fig. 7. The increase of the number of wells is accompanied by lower production rate of one well, though the total withdrawal capacity from UGS increases. The lower production rates were obtained for a higher minimum wellhead pressure values.

Another production parameter associated with the well production rate is 'reservoir depression' expressed as a difference of reservoir pressure and bottom pressure in the well $\left(p_{r}-p_{w}\right)$. The analysis of the plot in fig. 7 reveals that the highest production rate in a well is observed for a small number of production wells and low wellhead pressure value. In these conditions the reservoir pressure is lowest, therefore the reservoir depression is highest. The changes of this parameter in a function of the number of wells for two different values of wellhead pressure are shown in fig. 8. The depression with which the wells act on the reservoir is higher at the lower wellhead pressure, to decrease with the growing number of wells.

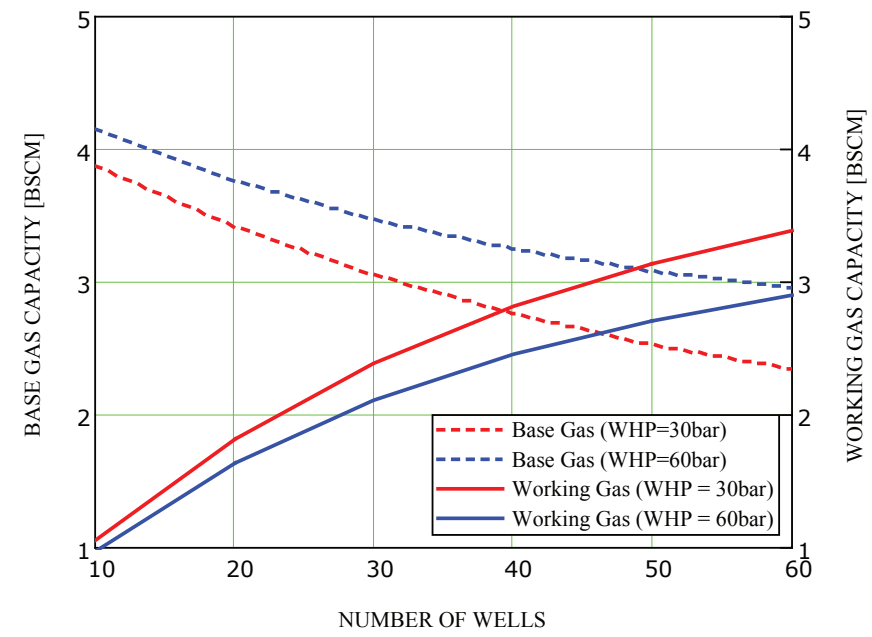

Fig. 5. Active and buffer capacity of UGS in a function of vertical wells number [own adaptation] 


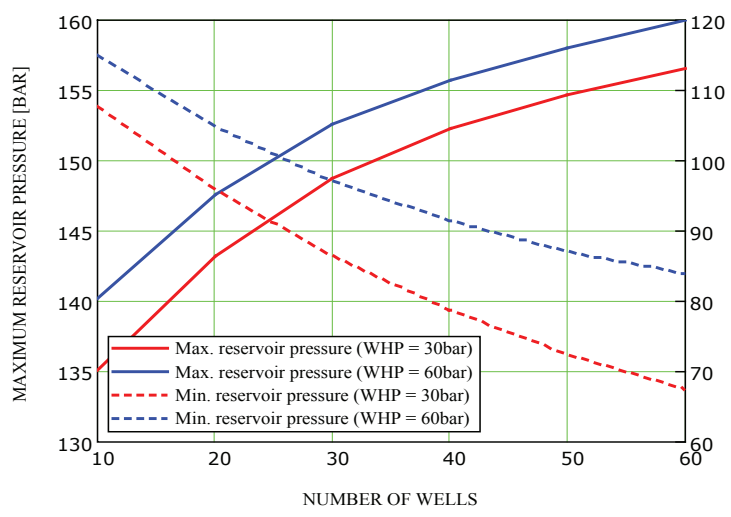

Fig. 6. Range of UGS pressures in a function of vertical wells number [own adaptation]

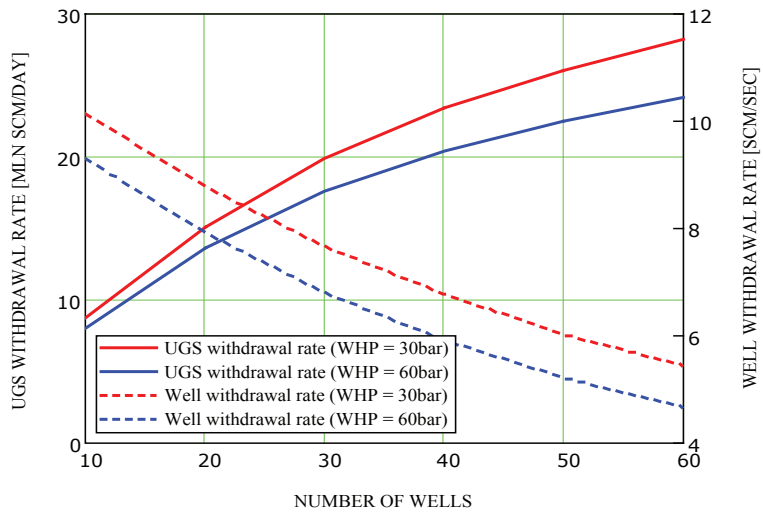

Fig. 7. Production rate of a well and summaric withdrawal rate of gas from UGS in a function of vertical wells number [own adaptation]

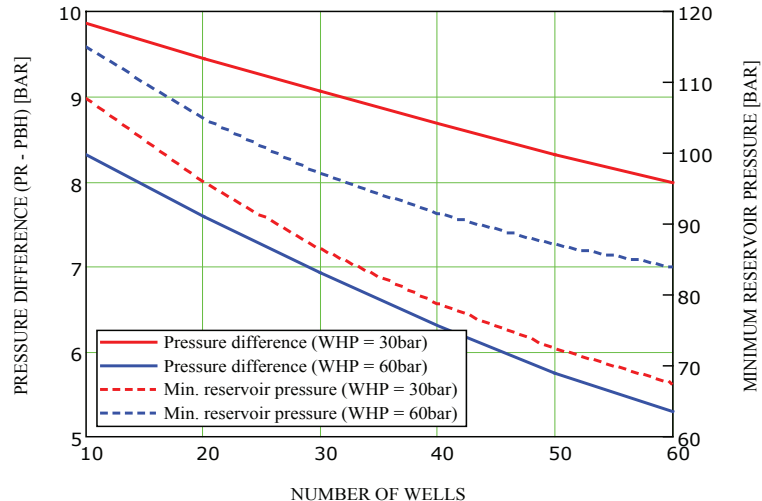

Fig. 8. Reservoir depression for vertical wells in a function of their number [own adaptation] 


\section{STORING WITH THE USE OF HORIZONTAL WELLS}

Analogous calculations of UGS production rate were made for horizontal wells. The results are presented in table 4 .

\section{Table 4}

Results of calculations for storages making use of horizontal wells

\begin{tabular}{|c|c|c|c|c|c|c|}
\hline Number of wells & 5 & 10 & 15 & 20 & 25 & 30 \\
\hline \multicolumn{7}{|l|}{ Wellhead Pressure 30 bar } \\
\hline Base Gas Capacity, [Bscm] & 3.72 & 3.16 & 2.73 & 2.42 & 2.19 & 2.0 \\
\hline Working Gas Capacity, [Bscm] & 1.34 & 2.24 & 2.86 & 3.30 & 3.63 & 3.84 \\
\hline Well Withdrawal Rate, $[\mathrm{Scm} / \mathrm{sec}]$ & 25.8 & 21.62 & 18.39 & 15.90 & 13.97 & 12.34 \\
\hline UGS Withdrawal Rate, [Mln Scm/d] & 11.14 & 18.73 & 23.83 & 27.47 & 30.18 & 32.0 \\
\hline Top Reservoir Pressure, [bar] & 138.5 & 147.5 & 152.9 & 156.1 & 158.8 & 159.6 \\
\hline Base Reservoir Pressure, [bar] & 103.6 & 88.97 & 77.91 & 69.59 & 63.36 & 58.27 \\
\hline Depression $\left(\mathrm{P}_{\mathrm{r}}-\mathrm{P}_{\mathrm{w}}\right),[\mathrm{bar}]$ & 1.9 & 1.81 & 1.74 & 1.66 & 1.60 & 1.53 \\
\hline \multicolumn{7}{|l|}{ Wellhead Pressure 60 bar } \\
\hline Base Gas Capacity, [Bscm] & 4.01 & 3.54 & 3.21 & 2.99 & 2.84 & 2.73 \\
\hline Working Gas Capacity, [Bscm] & 1.23 & 2.01 & 2.51 & 2.85 & 3.07 & 3.20 \\
\hline Well Withdrawal Rate, $[\mathrm{Scm} / \mathrm{sec}]$ & 23.71 & 19.40 & 16.15 & 13.74 & 11.86 & 10.36 \\
\hline UGS Withdrawal Rate, [Mln Scm/d] & 10.24 & 16.76 & 20.94 & 23.74 & 25.63 & 26.85 \\
\hline Top Reservoir Pressure, [bar] & 143.3 & 151.6 & 156.3 & 159.5 & 161.3 & 161.7 \\
\hline Base Reservoir Pressure, [bar] & 111.2 & 98.93 & 90.47 & 84.71 & 80.64 & 77.65 \\
\hline Depression $\left(\mathrm{P}_{\mathrm{r}}-\mathrm{P}_{\mathrm{w}}\right)$, [bar] & 1.64 & 1.47 & 1.32 & 1.19 & 1.07 & 0.96 \\
\hline
\end{tabular}

The course changes of UGS work parameters, i.e. active capacity, buffer capacity, UGS work pressure, well production rate and daily UGS withdrawal rates in a function of wells number are similar to those for vertical wells. The analysis of data in table 4 shows that the basic differences result from the number of wells needed for obtaining similar UGS work parameters. If gas is stored with the use of horizontal wells, their number is much smaller, which is directly connected with their higher productivity. Also the depression with which the wells act on the reservoir is much lower, and so the risk of water cones formation or other unfavorable effects, is lower.

The length of the horizontal section may have a significant influence on the efficiency of the horizontal well. Additional calculations were performed for the production rate of wells differing in the length of their horizontal sections, which were increased and decreased with respect to the assumed length of $500 \mathrm{~m}$. The obtained results showed to a slightly higher production rate of a well with longer horizontal section, which may stem from relatively high thickness of the reservoir and low heterogeneity of the porous medium.

The analysis of the results reveals that the active UGS capacity of $3.8 \mathrm{mld} \mathrm{Nm}^{3}$ can be obtained for 30 horizontal wells at a wellhead pressure of 30 bar. At the minimum wellhead 
pressure of 60 bar the active capacity was equal to $3.2 \mathrm{mld} \mathrm{Nm}^{3}$. However, the analysis of data in table 4 shows that the same active capacity can be obtained with the use of 15 to 20 horizontal wells at wellhead pressure of 30 bar.

The results of the analyses of horizontal and vertical wells indicate that depending on the number of applied production wells and also minimum wellhead pressure, it is also the UGS active and buffer capacity which changes, and consequently, the range of UGD work pressures as well. Higher UGS active capacity and a broader range of UGS work pressures were obtained at a lower wellhead pressure, which seems to be quite natural. However, the increase of the active capacity necessitates a higher number of wells and gas compression to a higher pressure when the UGS is being filled out.

The use of properly equipped and well designed horizontal wells should be an option thanks to which the number of wells can be reduced and flexible operation of UGS provided.

\section{CONCLUSIONS}

1. The presented methodics, based on analytical equations for gas flow in a reservoir and in a well, has been supplemented with mass balance equations, thanks to which the basic UGD work parameters, i.e. active capacity, buffer capacity, required number of horizontal/vertical wells, etc. could be relatively easily established with considerable scanty data.

2. The presented methodics can be used for preliminary evaluation of depleted gas fields as potential underground gas storages and for development of already existing UGS with the use of their downhole infrastructure and storage surface area.

3. The analyses of performed calculations reveals that an underground gas storage of 3 to 4 mld $\mathrm{Nm}^{3}$ capacity can be established in the analyzed reservoir, depending on the number of production wells and boundary conditions in the form of minimum and maximum reservoir and wellhead pressures.

4. The analysis of variant results reveals that the active capacity of UGS is considerably influenced not only by the structure, capacity and maximum pressure but also by way in which the reservoir was developed, extent to which the near-well zone was damaged (skin-effect), the design and equipment used in the production wells, number and type of wells, boundary conditions of wellhead pressures in gas injection/withdrawal cycle.

Keywords: underground gas storage, storages capacity, gas consumption, vertical and horizontal wells

\section{NOMENCLATURE}

$\mathrm{Pi} \quad-\quad$ initial reservoir pressure, $\mathrm{Pa}$

$\mathrm{p}_{\mathrm{r}} \quad-$ average reservoir pressure, $\mathrm{Pa}$

$\mathrm{p}_{\mathrm{w}}-$ bottom pressure in well, $\mathrm{Pa}$

$\mathrm{G} \quad-$ initial gas in place, $\mathrm{Nm}^{3}$ 
$\mathrm{Gp}$ - gas withdrawn from reservoir, $\mathrm{Nm}^{3}$

$\mathrm{Gz}$ - gas injected to reservoir, $\mathrm{Nm}^{3}$

$\mathrm{k} \quad-$ permeability, $\mathrm{m}^{2}$,

$\mathrm{k}_{\mathrm{H}} \quad-$ horizontal permeability, $\mathrm{m}^{2}$

$\mathrm{k}_{\mathrm{v}}-$ vertical permeability, $\mathrm{m}^{2}$

$\mathrm{h} \quad-$ thickness of reservoir, $\mathrm{m}$

$\mathrm{T} \quad-$ reservoir temperature, $\mathrm{K}$

$\mathrm{Z}$ - gas pseudo-compressibility factor,

$r_{e} \quad-$ well-range radius, $m$

$\mathrm{r}_{\mathrm{w}}-$ well radius, $\mathrm{m}$

$\mathrm{S}_{\mathrm{m}}-$ mechanical skin-effect,

$\mathrm{q}$ - well production rate, $\mathrm{Nm}^{3} / \mathrm{s}$

$D_{t}-$ coefficient of flow turbulence in reservoir, $d / \mathrm{mscf}$

$\mathrm{S}_{\mathrm{g}}-$ relative density of gas,

$\mu \quad-$ gas viscosity, $\mathrm{Pa} \cdot \mathrm{s}$

L - length of horizontal section of a horizontal well, $\mathrm{m}$

$\mathrm{R} \quad$ - individual gaseous constant, $\mathrm{J} / \mathrm{kg} \cdot \mathrm{K}$

$\mathrm{H} \quad-$ depth of well, $\mathrm{m}$

$\mathrm{D} \quad-\quad$ diameter of production wells, $\mathrm{m}$

$\lambda-$ coefficient of linear drag,

$\mathrm{r}_{\mathrm{wH}}-$ effective radius of horizontal well, $\mathrm{m}$

a $\quad-$ coefficient of bi-term equation, $\mathrm{Pa}^{2} \cdot \mathrm{s} / \mathrm{m}^{3}$

b $\quad-$ coefficient of bi-term equation, $\mathrm{Pa}^{2} \cdot \mathrm{s}^{2} / \mathrm{m}^{6}$

$\mathrm{ks}-$ permeability at near-well zone, $\mathrm{mD}$

$\mathrm{h}_{\text {perf }}-$ length of well's perforation, $\mathrm{ft}$,

$\beta_{\mathrm{H}}-$ non-Darcy flow coefficient, $1 / \mathrm{ft}$

\section{REFERENCES}

[1] Hagoort J., „Fundamentals of Gas Reservoir Engineering”, Elsevier -Amsterdam Oxford - New - York - Tokyo, 1988.

[2] Economides M.J., Hill A.D., „Petroleum Production Systems”, Prentice-Hall PTR, New Jersey 1994.

[3] Jahn F, Cook M. Graham, M.: „Hydrocarbon Exploration and Production” 2nd edition, Elsevier 2008r.

[4] Bergin S.R., Shikari Y.A., „A Horizontal Well in Gas Storage: A case Study”, SPE 26165, 1993r.

[5] Wang X., Economides M., „Advanced Natural Gas Engineering” Gulf Publishing Company, 2009r.

[6] Ahmed T, McKinney P.D., „Advanced Reservoir Engineering” Gulf Professional Publishing - imprint of Elsevier, 2005r. 
[7] McVay D.A, Spivey J.P., „Optimizing Gas Storage Reservoir Performance”, SPE 28639, 1994r.

[8] Tureyen O.I., Karaalioglu H, Sataman A., „Effect of the Well Conditions on the Performance of Underground Gas-Storage", SPE 59737, 2000r.

[9] Azin R. Nasiri A., Entezari J. A., Montazeri G.H., „Investigation of Underground Gas Storage in Partially Depleted Gas Reservoir", SPE 113588, 2008r.

[10] Dune J.W., „Gas Storage Field Development Optimization”, SPE 1503, 1967r. 